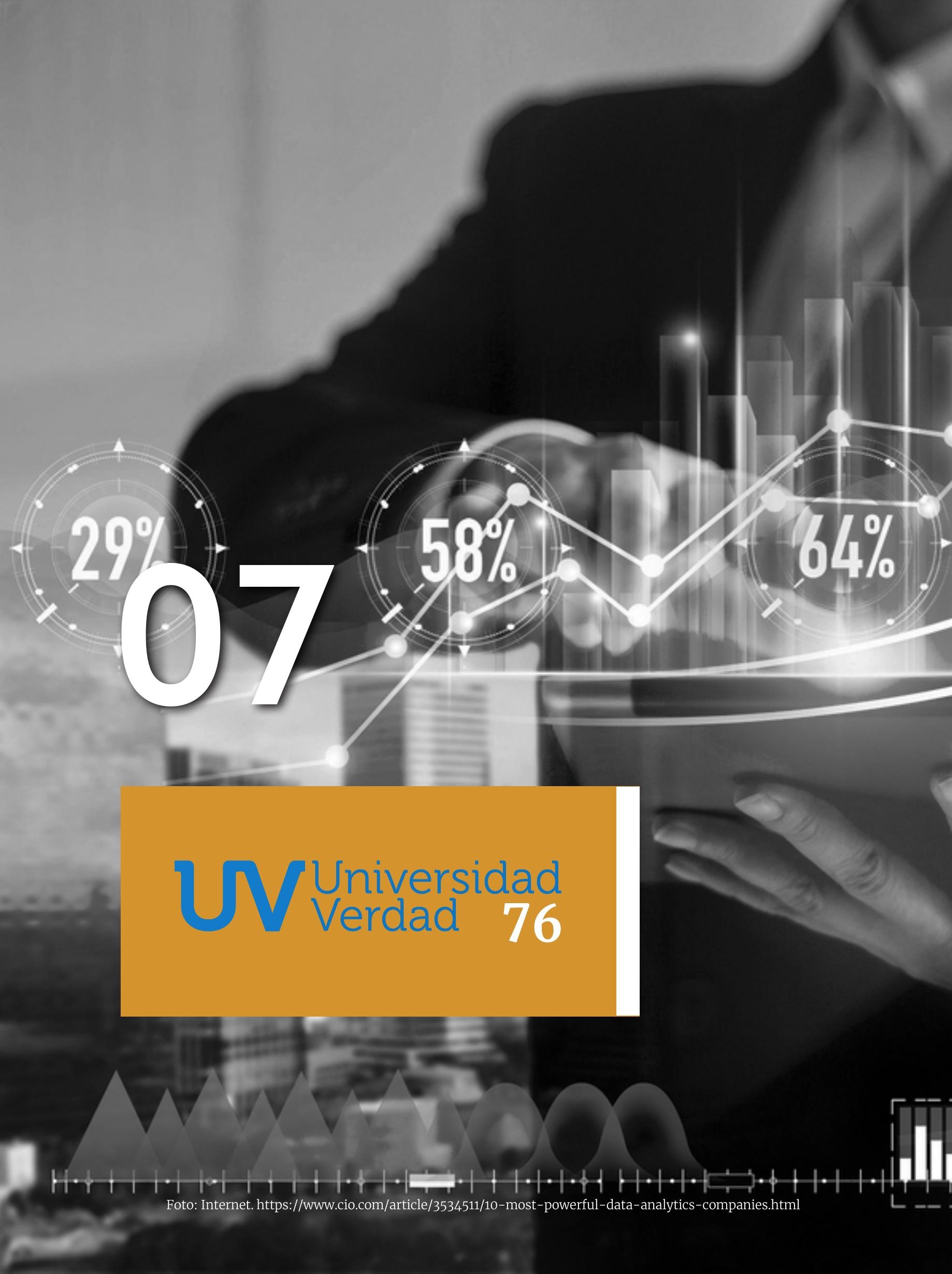




\title{
LAS NUEVAS TECNOLOGÍAS DE INFORMACIÓN Y COMUNICACIÓN A LA VANGUARDIA DEL SECTOR DEL TURISMO
}

\section{New Information and Communication Technologies at the forefront of the Tourism Sector}

\begin{abstract}
(iD) Mgt. Katherine Ortiz Vidal. Profesora de Diseño de Interfaz, Subdirectora Web en el Departamento de Tecnologías de la Información -Universidad del Azuay. UDA. (Ecuador) (kortiz@uazuay.edu.ec) (https://orcid.org/00o0-0003-4427-3927)
\end{abstract}

(iD Ing. Juan Carlos Pauta Ortiz, MBA. Profesor de Tecnologías de la Información, Mundo Electrónico, Universidad del Azuay.UDA (Ecuador) (jcpauta@uazuay.edu.ec) (https:// orcid.org/0000-0002-9521-7434)

\section{Resumen}

El valor actual que adquiere la información a nivel mundial y el interés por conseguirla hace posible que podamos interactuar con ella, obtenerla y analizarla según nuestras expectativas. Esta propuesta investigativa tiene como objetivo identificar el impacto que tienen las Nuevas Tecnologías de Información y la Comunicación (NTICs) en el sector del turismo, alrededor del mundo; e identificar la forma de tratar la información, con el fin de encontrar resultados que favorezcan a la toma de decisiones empresariales. Se darán a conocer, además, las formas de hacer turismo con realidad virtual, las formas de obtener información al estar físicamente en un lugar y el aporte de conocimiento que se obtiene con la realidad aumentada. Para este fin, se considera el concepto de viajero actual y la interacción que tiene con el internet y con los dispositivos inteligentes.
La investigación es de alcance descriptivo relacional. Para realizar el estudio se utilizaron herramientas informáticas y se obtuvieron no solo resultados, sino gráficas que permiten complementar la información. Otra parte de los resultados se generaron a través de scraping web y fueron posteriormente comparados, con el fin de utilizar la herramienta informática como medio para predecir el comportamiento de la data histórica obtenida a través del modelo de regresión lineal simple.

\section{Abstract \\ The current value that information acquires worldwide and the interest in obtaining it makes it possible for us to interact with it, obtain it and analyze it according to our interests. This research proposal aims to identify the impact that New Information and Communication Technologies (ICTs) have on the tourism sector around the world and the way of treating information to find results}


that favor business decision-making. In this article, it is also about knowing the ways of doing tourism with virtual reality, the ways of obtaining information by being physically in the place and the contribution of knowledge that is obtained with augmented reality. It is also considered the conception of the current traveler and the interaction it has with the internet and with smart devices.

\section{Palabras clave}

NTICS, Aprendizaje automático, Scraping, Análisis de datos, GoogleTrends, Turismo.

\section{Keywords}

NTICS, Machine learning, Scraping, Data analysis, GoogleTrends, Tourism.
This research of descriptive relational scope allowed obtaining and analyzing information with computer tools that, in addition to their results on the issues raised, generate graphs and complement results related to the topic. These results were also generated through web scraping and comparisons, finally, after reviewing the bibliography, use the computer tool that allows predicting the behavior of the historical data obtained through the simple linear regression model.
1.

\section{Introducción}

El avance vertiginoso de la tecnología en esta nueva era, considerada como sociedad del conocimiento (Sánchez, 2016), ha dado lugar a que, los bienes y servicios que el hombre requiere para satisfacer sus necesidades se consigan de diferentes formas en el mercado. En el sector turístico, quienes planean una nueva aventura de placer o negocios lo hacen aprovechando la conexión mundial. Hoy, se los conoce como los nuevos viajeros o viajeros del siglo XXI ( $\mathrm{La}$ Vanguardia, 2015). Estos viajeros, con antelación, planifican y hasta personalizan sus destinos; lo que representa, en muchas ocasiones, la alternativa perfecta, pues les permite escoger la mejor opción en relación con el costo, el destino, estancias, traslados y promociones en tickets, para su viaje o cualquier evento complementario.

Las empresas proveedoras de servicios de turismo deben buscar e innovar sus propuestas, tomar acciones ofensivas o defensivas, para crear una posición estratégica en una industria, con la finalidad de hacer frente, con éxito, a las fuerzas competitivas y así generar un retorno sobre la inversión, y una ventaja competitiva (Porter, 1991). De esta manera, las empresas resultan atractivas para sus clientes. Estas innovaciones radican en conocer a sus clientes, sus preferencias y hasta sus necesidades; para esto, hoy en día pueden contar con amplia información, pues cada rastro de búsqueda o publicación en internet se registra. Actualmente, esta es considerada una información de mucho valor (Fernández, 2012) que, a través de herramientas o aplicaciones informáticas, se puede analizar, con la finalidad de encontrar patrones de comportamiento que permitan mejorar la calidad de servicios al turista. De esta manera, los proveedores de servicios turísticos obtendrían una posible ventaja competitiva, lo que les permitiría mejorar sus estrategias.

En este estudio se revisarán las posibilidades que nos ofrece la utilización de las nuevas tecnologías de información y comunicación (NTICs) a través de la bibliografía existente. Además, la información que 
se obtenga se relacionará con los distintos temas que interesan a este estudio. A través del uso de estas tecnologías, y la información que obtengamos de bases de datos, en su mayoría de libre acceso, encontraremos nuevas formas de hacer turismo, de hacer negocios y de innovar. Lo que pretendemos demostrar es cómo, a través de la navegación por la red y el uso de información almacenada, se puede llegar tan lejos como se desea.

\subsection{Conectividad y turismo}

Hoy en día, con la evolución de las tecnologías de información es posible, por un lado, tomar la decisión que nos lleve a conocer los lugares más recónditos de nuestro planeta sin desplazarnos, y, por otro lado, desplazarnos y, a la vez, recibir información a detalle de lo que en casa sucede.

Es innegable el auge cada vez mayor de las tecnologías de la información y comunicación. Estas han dado lugar a que la sociedad, a escala mundial, dependa de su uso; desde la era digital, hasta la sociedad actual de la información y del conocimiento, en la que, figurativamente, se menciona que "todo" se encuentra en la red. La nueva revolución digital consiste en el cambio de la internet del consumo a la internet de la producción, con un alcance insospechado (CEPAL, 2016).

Esta revolución impacta en la forma de hacer empresa, en todos los ámbitos. Los cambios en el mercado implican nuevas dinámicas empresariales. Es preciso innovar en procesos tanto internos, como externos, que se enmarquen en la demanda y en la velocidad a la que esta evoluciona. La empresa debe ser flexible y adaptable, de forma que en sus planificaciones se incremente la importancia de la incursión en las NTIC. La preparación y propuestas serán las que le permitan permanecer y competir en esta nueva era humana. Cabe recalcar que en este proceso tienen espacio las micro, pequeñas y medianas empresas; posiblemente, la rápida adaptabilidad a las necesidades del mercado sea su gran fortaleza. Sin embargo, las grandes empresas serán las que defiendan sus intereses con sus potencialidades, experiencia, planificación y objetivos, características de su tamaño, y se enfoquen en las necesidades de su sector; es decir, que su propia estructura les exigirá innovar.
"En el siglo XXI, solo hay dos tipos de negocios: Los que están presentes en internet $\mathrm{y}$, los que no existen, (Bill Gates)" (citado en Evolutionmrk, 2014, párr. 2). Esta pudo haber sido una predicción muy alejada de la realidad, pero la visión parece ser cierta; hoy en día, las empresas, para hacer negocios, deben tener su presencia en internet, aunque existan algunas excepciones. Sin embargo, se debe tener en cuenta que el sector turístico adoptó el internet de manera inmediata, por las potencialidades que el nuevo sistema ofrece.

El internet, en el área del turismo, es sin duda, el medio ideal, pues ofrece accesibilidad, comodidad, rapidez, novedad, cobertura, riqueza, flexibilidad y bajo coste de implementación. Es necesario hacer énfasis en la seguridad que brinda a los usuarios para realizar transacciones en línea, en el cumplimiento de las ofertas, la personalización de los servicios, y otros parámetros. Todo ello, se enmarca en el concepto de $e$-tourism que incluye el diseño, la implementación y la aplicación de las TIC y soluciones de comercio electrónico en la industria del turismo, el análisis del impacto de los procesos económicos y técnicos, así como las estructuras del mercado de todos los actores involucrados en las experiencias de los viajeros (Sánchez et ál., 2018). Causado et ál., (2015), mencionan que las TIC también son utilizadas por las empresas turísticas para ampliar sus operaciones, gestionar su inventario y maximizar su rentabilidad.

En la actualidad, la internet forma parte de la vida de las empresas y las personas; ofrece alternativas a diferentes estilos de vida, más aún, cuando la ONU aprobó una resolución que reconoce el acceso a internet como uno de los derechos humanos e insta a sus miembros a que actúen contra la exclusión digital (Siglo XXI, 2019). Cada país perteneciente a la ONU, se propuso ofrecer las mejores alternativas que disminuyan la brecha digital. Hace 20 años, tan solo el $4 \%$ de población mundial tenía acceso, para el año 2019 esta cifra incrementó a 58.8\%, cifra que representa 84 millones de internautas con acceso. El Diario Siglo XXI (2019) considera que es un reto para el presupuesto de las familias de hoy acceder a este servicio, debido al costo que se tiene que pagar por el servicio. Según los datos de la plataforma Speedtest.net, la velocidad promedio mundial es de 70Mb/ seg (We are social, 2020). Por otra parte, países del 
primer mundo superan este ancho de banda y países del tercer mundo posiblemente lleguen a tener el mismo servicio en un tiempo no muy lejano. Se debe considerar que el precio por el servicio cambiará, ya que se incrementará la velocidad $\mathrm{y}$, por tanto, mejorará el precio, como parte de la política pública en beneficio de la sociedad (MINTEL, 2015). Sin lugar a duda, la posibilidad de tener mejoras en la conexión a internet permitirá un mayor acceso a los servicios, a través de dispositivos con tecnología cada vez más avanzada.

En la cotidianidad, se ha incrementado la relación con las NTICs. Es común llamarles por su nombre Alexa, Siri, Cartona o Google Now, estas cumplen el rol de un asistente personal o familiar. Es lo que las empresas Amazon, Apple, Microsoft o Google respectivamente ofrecen a sus fieles seguidores, a la fecha, han desarrollado aplicaciones que registran y aprenden a través de las peticiones diarias, asimismo, trabajan sin horario y predicen los requerimientos incluso antes de ser consultadas; podrían incrementar sus actividades en el hogar, hasta ser parte de él. Fedele (2011) indica que estos asistentes se basan en los datos de internet, en su ubicación y en el tono de voz con el que se les despierte; funcionan como compañeros de viaje y son una muy buena alternativa, pues, con el solo hecho de activar su localización pueden reconocer de manera inmediata lugares, mapas, restaurantes, hospedajes, estaciones de combustible, entre otros. Además, tienen la posibilidad de interactuar con muchos dispositivos, sin que estos estén en el mismo lugar, forman redes de objetos, son capaces de coleccionar e intercambiar datos si se habilitan los dispositivos con los que van a interactuar, colaborar y aprender. Todo esto se denomina hoy en día como El Internet de las Cosas "IoT" -Internet of Things- (Conecta Software, 2018).

Las Nuevas Tecnologías de la Información y Comunicación (NTICs) se refieren también a las nuevas formas del tratamiento de la información y su comunicación. En el área del turismo, estas tienen su aporte significativo, ya que dan respuesta al perfil del viajero social del siglo XXI, quien se orienta en la búsqueda de "experiencias y sensaciones", da valor a emociones y sentimientos. Es decir, son viajeros que acumulan datos, fotografías, videos, interacciones, que son parte de su historia, y actualmente se los conoce como parte de la Dream Society (Arroyo, 2011).
Estas nuevas tecnologías ofrecen la personalización de la experiencia. El uso de plataformas digitales brinda alternativas más atractivas, pues los resultados se basan en comparativas entre diferentes sitios web que ofrecen distintos lugares, hospedajes, traslados, servicios, itinerarios, y más, con la opción de encontrar los mejores precios; lo que provoca la dinamización de la economía del sector.

Hacer turismo desde casa podría considerarse la respuesta a la inquietud inicial. Cuando no es posible el desplazamiento físico para hacer turismo, encontrar museos, parques arqueológicos o monumentos históricamente destacados, la realidad virtual, la fotogrametría y la digitalización nos permiten ser parte de la experiencia inmersa, a través del uso de Nuevas Tecnologías de Información y Comunicación. La visita al Museo Sefardi de Toledo es uno de los ejemplos. Ofrece una visita virtual por todo su campus, ofrece un video de 360 grados completamente interactivo, dispone 9 salas que recogen la colección permanente y $450 \mathrm{~m}^{2}$ para hacer la visita, se precisa tan solo de conexión a internet (DIDACTALIA, 2014). Describing Egypt es otro sitio en el que se puede hacer turismo digital, disfrutar de la historia de Egipto, conocer templos y tumbas de faraones con una experiencia de realidad virtual inmersiva; este es un ejemplo de sitios que en la actualidad son considerados inaccesibles, cerrados para su conservación o son sujeto de estudio como el proyecto: "Visita a la tumba del Faraón Ramses VI en el Valle de los Reyes" (Describing Egypt, 2015).

Por otra parte, la información física, sumada a los elementos digitales recibidos por un dispositivo con conexión a internet, se considera como realidad aumentada; crea una realidad mixta y permite disponer de más información de la que físicamente se obtendría (IONOS, 2019). Dar más información y de gran valor al viajero es lo que permite la aplicación de las tecnologías y el aumento de posibilidades en el sector de turismo. Ofertar guías turísticas interactivas a través de los dispositivos móviles consiste en encuadrar la imagen para que la información adicional sea presentada; permite que la visita sea más divertida, interactiva y con mayor cantidad de información. El turista, además, puede etiquetar imágenes, sonido, recomendaciones, etc., y compartir su experiencia con otros viajeros como menciona la revista (TYSMagazine, 2015). Los visitantes sienten un valor mayor 
con el contenido de la realidad aumentada, que con el docente que da vida al lugar; consideran que pueden revisar el material a su propio ritmo, retienen más cuando leen que cuando escuchan, y otros visitantes pueden observar su experiencia completa y acceder a ella (Almenara et ál., 2018).

Hacer público o presumir de las últimas vacaciones es la manera de compartir experiencias con el mundo. Cada día son más los viajeros que hacen esto, a través de redes sociales. Actualmente, Facebook e Instagram son las más utilizadas y las que mayor actividad de registro de datos poseen (We are social, 2020). Esto nos lleva a considerar que en ellas encontraremos incluso recomendaciones para nuestro próximo destino. Los usuarios permanecen conectados y, muchos de ellos, responden a las inquietudes como parte de su objetivo de publicación y de ser parte de una comunidad afín a sus gustos y preferencias hasta provocar el engagement, como compromiso de marca para sus seguidores y su interacción en la red (Duro, 2017).

Global Digital Report, realizó un estudio digital en 2019, en él publica que las redes sociales ya cuentan con 3.500 millones de usuarios, el 65\% tiene cuentas de Facebook, y el 96\% de ellos utiliza sus smarthphones y han compartido múltiples intereses personales y profesionales. Por esta razón, Facebook se ha convertido en la principal red social de turismo. Instagram tampoco se detiene, desde el año 2012, cuando Facebook la adquirió, registra 1.000 millones de seguidores, por lo que puede llegar a convertirse en una fuerte competencia dentro del grupo de redes sociales (We are social, 2020).Todo ello dependerá del comportamiento y las interacciones que tengan las empresas con los clientes.

\section{2.}

\section{Metodología}

Esta investigación, de alcance descriptivo relacional, busca obtener información que demuestre el impacto que han tenido las Nuevas Tecnologías de la Información y Comunicación (NTICs) en el sector del turismo. En primer lugar, mediante la revisión bibliográfica, se analizó el valor que adquiere la información con relación a las características del viajero actual y las nuevas formas de ofrecer turismo a través de la realidad virtual y realidad aumentada. En segundo lugar, se utilizó GoogleTrends como herramienta informática de libre acceso, para analizar la información, producto del rastro registrado por las búsquedas realizadas por usuarios en internet. Luego, se procedió a obtener información de sitios web mediante la simulación de la navegación humana y comprobar los resultados. Finalmente, se realizó una simulación para determinar el comportamiento de datos futuros, para esto fue necesario recurrir a información de años anteriores, determinar cuál fue la relación entre las búsquedas o variables, y con base en estos datos, pronosticar el comportamiento de las búsquedas que podrían registrarse en los próximos años.

Para lograr este objetivo, fue necesaria la aplicación de algoritmos de Machine Learning supervisados, que ejecutan el modelo de regresión lineal simple, en los que se buscó la tendencia para la predicción. Además, medimos el error con respecto a los puntos de entrada y el valor " $Y$ " de salida real a través de la herramienta informática Python (Pedregosa et ál., 2019), enfoque que se considera de carácter cuantitativo.

\section{3.}

\section{Resultados y conclusiones}

En la actualidad, se dispone de varias herramientas que proveen información con base en las interacciones registradas entre los usuarios de internet, tal es el caso de Google Trends, considerada como una herramienta de acceso libre, que permite comparar la popularidad de un término de búsqueda de una o más palabras o frases. De esta manera es posible conocer el nivel de búsqueda de un determinado keyword en un período de tiempo, pues la herramienta exhibe gráficamente las variaciones en valores relativos entre 0 y 100; 100 representa el valor más alto del nivel buscado de la keyword.

Las comparaciones se realizaron con información desde el año 2004 hasta la actualidad, lo que permitió ver los patrones y el comportamiento de un determinado sector o zona geográfica (ENyD, 2014). 


\section{Figura 1}

Comparación de búsquedas en Turismo Ecuador
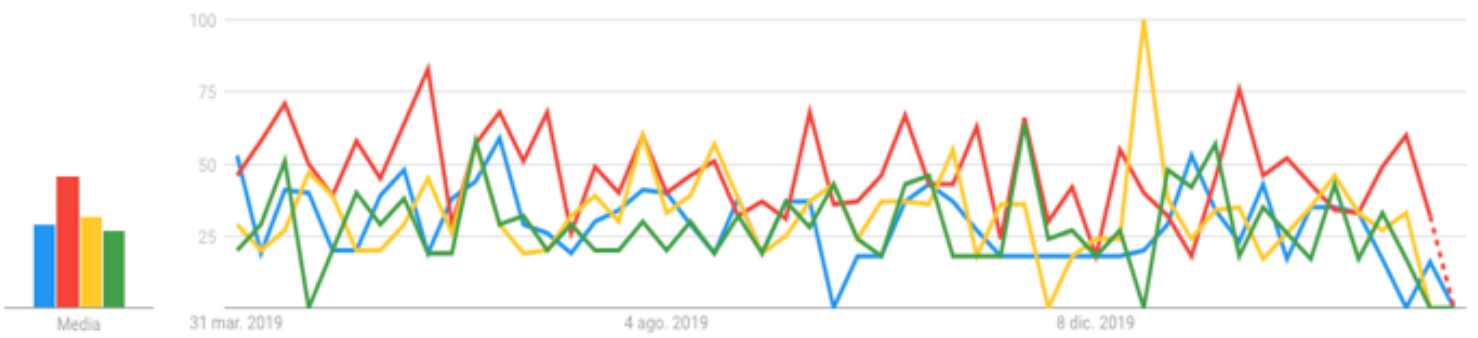

Fuente: Google Trends. [https://bit.ly/2VOYKny]

Las herramientas utilizadas ofrecen, además, la posibilidad, a través de mapas de calor, de distinguir las búsquedas en diferentes regiones de cada país; relaciona la búsqueda con el término que muestre un mayor crecimiento $\mathrm{y}$, de esta manera, permite apreciar de manera comparativa el comportamiento de hasta 5 términos y las relaciones entre ellos. Todo esto es posible por la cantidad de información que subimos a internet y que deja un rastro, nuestra huella digital; desde nuestra forma de navegar, hasta la geolocalización de nuestro smarthphone, el lugar donde nos encontramos, hasta qué lugares vistamos (González, 2018).

Conocer el interés del viajero social del siglo XXI da lugar a planificar y tomar decisiones para un futuro no muy lejano; para ello, se procedió a ejemplificar este análisis mediante el uso de la herramienta Google Trends. Se utilizó la información que esta herramienta provee acerca de registros de búsquedas que se relacionan a lugares del Ecuador. Los resultados de las búsquedas y la comparación referente a lugares turísticos durante el último año entre Quito (rojo), Guayaquil (amarillo), Cuenca (azul) y Galápagos (verde) expresan el comportamiento de navegación de los últimos 12 meses. Como resultado se obtuvo que "Quito-Ecuador-Turismo" es lo más representativo en cuanto a búsqueda semanal, comparado con Guayaquil, Cuenca y Galápagos, como lo muestra la Figura 1.

En resumen, se puede mencionar que la herramienta GoogleTrends analiza la información registrada y la presenta en períodos de tiempo semanales. La co- lumna Mayor valor corresponde a las semanas más buscadas, y la columna Menor valor corresponde a las semanas de menor registro de búsqueda. Registro que se da en relación a cada término buscado, sobre los datos tomados del último año y se resume en la Tabla 1. 


\section{Tabla 1}

Fechas con mayor y menor impacto de búsquedas

\begin{tabular}{|c|c|c|}
\hline Término buscado & Mayor valor & Menor valor \\
\hline \multirow{2}{*}{ Quito-ecuador-turismo } & 26 may - 1 jun & 8 dic -14 dic \\
\hline & 19 ene -25 ene & 5 ene - 11 ene \\
\hline Guayaquil-ecuador-turismo & 22 dic -28 dic & $24 \mathrm{nov}-30 \mathrm{nov}$ \\
\hline \multirow{2}{*}{ Cuenca-ecuador-turismo } & 16 jun -22 jun & 22 sep - 28 sep \\
\hline & 5 ene - 11 ene & 8 mar -14 mar \\
\hline \multirow{3}{*}{ Galápagos-ecuador-turismo } & 9 jun -15 jun & $21 \mathrm{abr}-27 \mathrm{abr}$ \\
\hline & 17 nov -23 nov & 22 dic -28 dic \\
\hline & & 8 mar - 14 mar \\
\hline
\end{tabular}

Fuente: GoogleTrends [https://bit.ly/3bPTyFn]

Otro ejemplo llevado a cabo corresponde a la búsqueda de términos relacionados con lugares turísticos mundialmente reconocidos como: Orlando, Torre Eiffel, Cancún, Vaticano. Posteriormente, esto fue relacionado con un término que representa un lugar turístico del Ecuador como Galápagos; el resultado obtenido expresado en la Figura 2 indica que el término Orlando es el que más registros de búsqueda obtiene desde el año 2004 hasta marzo de 2020. El término Vaticano presentó un incremento de búsquedas en el año 2005, en relación a los otros términos. Además, Cancún resultó mejor posicionado que los términos Torre Eifell y Galápagos en el mismo rango de fechas, información generada en GoogleTrends.

\section{Figura 2}

Comparación de búsquedas en el mundo (lugares turísticos)
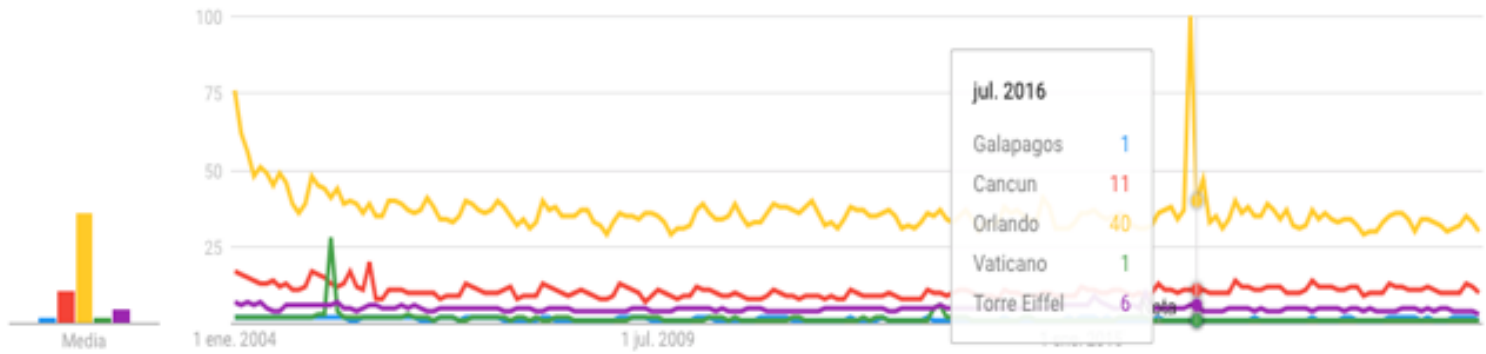

Fuente: GoogleTrends [https://bit.ly/2YhktWQ] 
Cabe mencionar que parte de los resultados que presenta esta herramienta GoogleTrends corresponde a un mapa de calor, que hace referencia a una lista ordenada de países de los en los que se registraron las búsquedas, relacionadas al término buscado, como lo expresa la Figura 3, en relación al término Galápagos.

\section{Figura 3}

Mapa de calor de los lugares registrados que buscan el término Galápagos
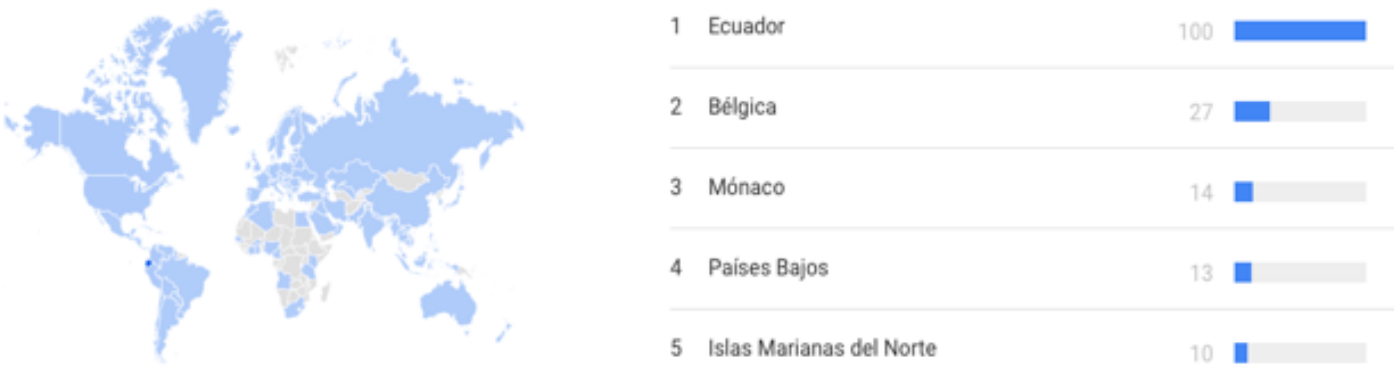

Fuente: GoogleTrends [ https://bit.ly/35lJ5z9]

Adicionalmente, GoogleTrends presenta una lista de términos relacionados al buscado. Es necesario indicar que la información registrada por Google se obtiene de diferentes fuentes que corresponden al propio motor de búsqueda, en las que se considera el buscador web, "google imágenes", "google videos" (youtube), "google shopping" y "google noticas". Las consultas relacionadas pueden contener palabras en diferentes idiomas, todo dependerá de cómo y en qué lugar fue registrada la búsqueda. La Figura 4 es el resultado de las consultas relacionadas a la búsqueda del término Galápagos.

\section{Figura 4}

Consultas relacionadas al término buscado: Galápagos

\begin{tabular}{lll} 
Consultas relacionadas (?) & En aumento & Aumento puntual \\
\hline 1 & koers galapagos & Aumento puntual \\
\hline 2 galapagos time & Aumento puntual \\
\hline 3 isabela galapagos & Aumento puntual \\
\hline 4 & nasdaq galapagos & \\
\hline 5 & galapagos wikipedia & Aumento puntual
\end{tabular}




\section{Figura 5}

Scraping lugares turísticos GoogleTrends con Python

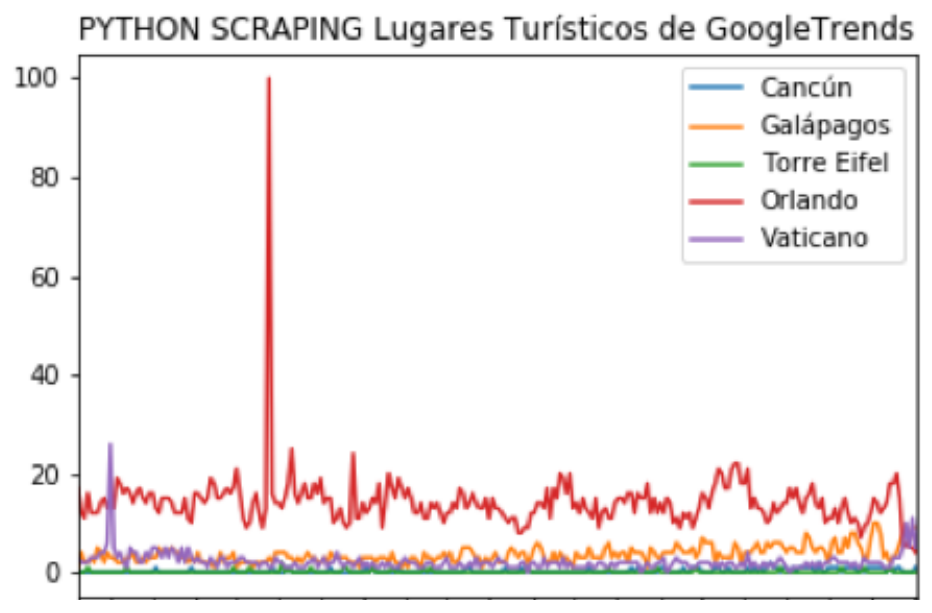

Consideramos importante acotar que, sobre la información que se obtiene resultado de las comparaciones realizadas, pueden utilizarse herramientas que permitan procesar y hacer análisis más complejos, entre ellas: Lenguaje R, Power Bi, Apache Spark, Apache Storm, MongoDB, entre otras (IIC, 2016). Python también es una de estas herramientas, la cual es considerada como un lenguaje de programación dinámico de propósito general (Desarrollo Web, 2003), se utiliza para la creación de un sitio web, para obtener información de otros sitios web, simulando navegación humana(scraping) y para la ciencia de datos (Siteground, 2020).

Para el ejemplo de simular la navegación humana o Scraping, se consideró la información obtenida en la Figura $1 \mathrm{y}$ fue necesaria la programación en Python. Con la ayuda de módulos adicionales de aplicaciones para la extracción de datos API (Aplication Programming Interface), se obtuvo la información de los últimos 5 años. Los resultados son semejantes, la base de datos es la misma, el propósito planteado de aplicar la técnica mediante software para obtener información de GoogleTrends simulando navegación humana, sobre un propósito definido, fue comprobada. En este caso, se consideró la información ya obtenida sobre búsquedas de términos que corresponden a sitios turísticos previamente encontrados, véase Figura 5.

Utilizando la misma herramienta se trató de ejemplificar el aprendizaje automático o machine learning, entendiendo este como la disciplina científica del ámbito de la inteligencia artificial que relaciona al "usuario", con la "tarea" y con la "máquina". Es importante mencionar que estos programas mejoran de forma autónoma con el paso del tiempo, sin la intervención humana (González, 2018).

Para realizar el ejemplo sobre la tendencia de predicción en Python fue necesario utilizar algoritmos que permiten identificar patrones complejos, aquellos que tienen la capacidad de predecir comportamientos futuros.

En el área de turismo, aplicar la predicción con regresión lineal simple nos permitió obtener como resultado el comportamiento de búsquedas futuras, aplicado a la información resultante de GoogleTrends, sobre términos correspondientes a países como destinos turísticos, entre los que se consideraron: Ecuador, Perú, Colombia, Venezuela y Chile. El resultado, producto de este análisis, fue interpretado en un plano 3D, resultado que expresa un comportamiento futuro similar a su registro de años anteriores. La excepción se dio en el caso Ecuador-Venezuela, hace un par de años atrás cuando el término Ecuador superó en búsquedas al término Venezuela, pero se mantiene por debajo de las búsquedas que se realizarán con respecto a los términos Perú, Chile y Colombia, como se muestra en la Figura 6. 


\section{Figura 6}

Predicción con regresión lineal en Python: Países como destinos turísticos

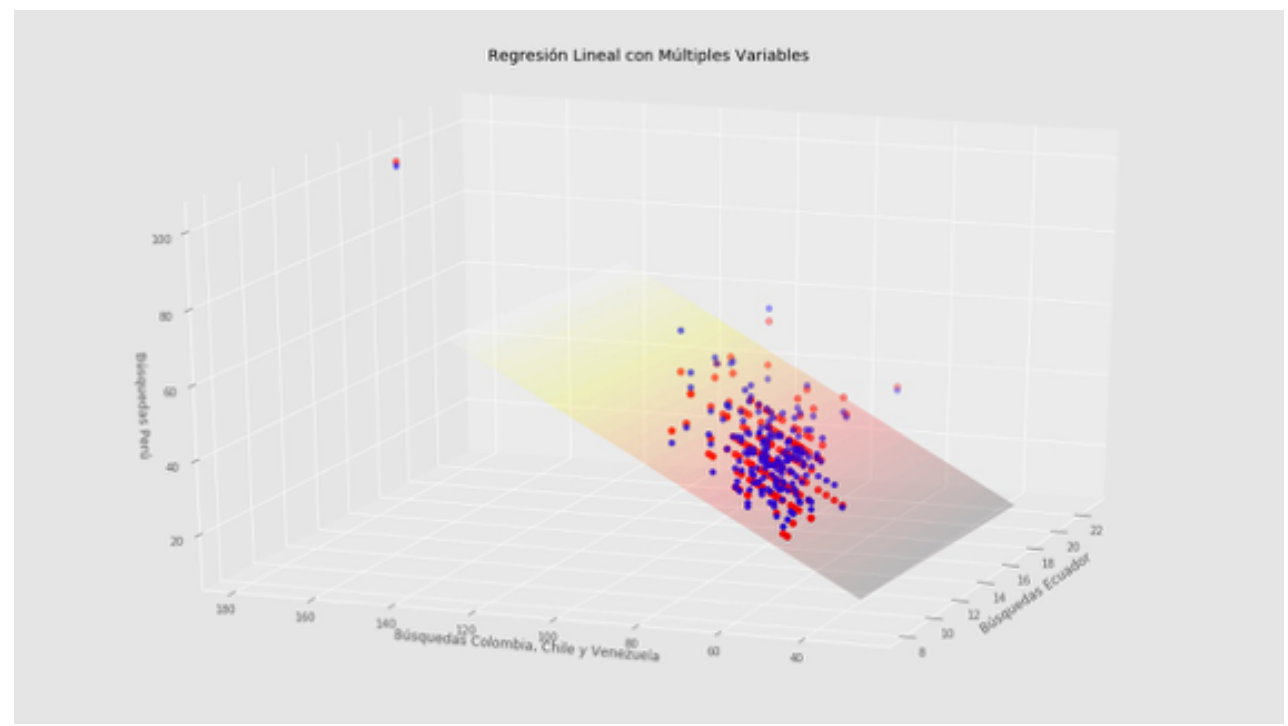

Fuente: Elaboración propia con datos obtenidos de GoogleTrendsen Python

En el caso específico de un negocio relacionado al sector de turismo, la aplicación de estas herramientas significaría la personalización del servicio sobre sus clientes. El resultado del análisis de la información permitiría, entre otros, predecir la nacionalidad de los turistas en determinadas épocas del año, saber con antelación si una reserva es sensible a ser cancelada, predecir retrasos de vuelos, identificar patrones de comportamiento de clientes, necesidades de huéspedes, entre otras; resultados que pueden aportar a la toma de decisiones, para alcanzar los objetivos planteados.

El resultado más importante que se ha conseguido a través de este trabajo es conocer, planificar y satisfacer las necesidades con antelación. El Diario de Alcalá (2019) publicó que el Machine Learning está cada vez más enlazado al sector turismo. Es hora entonces de poner atención, replantear objetivos y relacionar información de clientes y su satisfacción. Conseguir resultados nuevos y oportunos podría ofrecernos la posibilidad de ser efectivos, sobre una demanda cada vez más exigente y competitiva.

\section{Referencias}

Almenara, J., Vázquez, E., \& López, E. (2018). Uso de la realidad aumentada como recurso didáctico en la enseñanza universitaria. Formación Universitaria, 11(1), 25-34. https://bit.ly/2UHoLBr

Arroyo, R. (2011). La Sociedad de ensueño del turismo. Anuario Turismo y Sociedad, (12), 17-26.

Causado, E., García, J., Martínez, J., \& Herrera, A. (2015). Tecnologías de información y comunicación. http://hdl.handle.net/11323/3184

CEPAL. (2016). La nueva revolución digital. De la internet del consumo a la internet de la producción. ELAC 2018 La Revolucion Digital. Santiago.

Conecta Software (2018). ¿Que es el Internet de las cosas? ConectaSoftware.com. Proyecto Cofinanciado por el Fondo Europeo de Desarrollo Regional. https://bit.ly/2BevVJz 
Desarrollo Web. (2003). Qué es Python. https://desarrolloweb.com/articulos/1325.php

Describing Egypt. (2015). Visita a la tumba del Faraón Ramses VI en el Valle de los Reyes. https://describingegypt.com/

DIDACTALIA. (2014). Visita Virtual del Museo Sefardí de Toledo. https://bit.ly/2ZKNoot

Duro, S. (2017). (6 de marzo de 2017) ¡Cómo generar engagement en redes sociales humanizando tu marca! (Blog: SEMRUSH.com).

https://bit.ly/3gw3ExY

El Diario de Alcalá. (2019). Machine Learning: cada vez más enlazado con el sector turismo.

https://bit.ly/3ervny3

ENyD (Escuela de Negocios y Dirección). (12 de marzo de 2014). Qué es y para qué sirve Google Trends. https://bit.ly/2yFsF8W

Evolutionmrk. (2014). (Blog) evolutionmrk.wordpress.com. Marketing E-Evolution.

https://bit.ly/2XCLemK

Fedele L. (2011) From Basic Maintenance to Advanced Maintenance. In: Methodologies and Techniques for Advanced Maintenance. Springer, London.

https://doi.org/10.1007/978-0-85729-103-5_5

Fernández, J. (2012). (5 de febrero del 2012). La huella imborrable. El Periódico. El rastro digital. 4-7. https://bit.ly/2X9Mnmx

González, S. (18 de septiembre de 2018). Cuidado con el rastro que dejas en internet. El Mundo. https://bit.ly/3extSP3

Instituto de Ingeniería y Conocimiento (IIC). (13 de octubre de 2016). 7 herramientas Big Data para tu empresa. https://bit.ly/2M8VIF6
IONOS (2019) Más allá de Picachú: realidad aumentada en eCommerce. https://bit.ly/2XCK5eW

La Vanguardia. (2015). (16 de noviembre de 2015). ¿Cuál es el perfil del viajero del siglo XXI? Magazine viajes. https://bit.ly/3cbW2xx

Ministerio de Telecomunicaciones (MINTEL). (2015). Plan Nacional de Telecomunicaciones. https://bit.ly/3gw4wme

Porter, M. (1991). Ventaja competitiva. México. Grupo Patria Cultural. Alay ediciones.

Pedregosa, et ál. (2011) Scikit-learn: Machine Learning in Python, JMLR 12, 2825-2830.

https://bit.ly/360WKjf

Sánchez, A. (2016). La Sociedad de la Información, Sociedad del Conocimiento y Sociedad del Aprendizaje. Referentes en torno a su formación. Bibliotecas. Anales de Investigación, 12(2), 235-243.

Sánchez, M. Á., Fernández, M. T., Mier-Terán., F. (2018). Revisión teórica de la relevancia de las nuevas tecnologías de la comunicación (TIC) en el sector turístico. Revista Turydes: Turismo y Desarrollo, (24). https://bit.ly/3daVbOF

Siglo XXI. (2019). (18 de diciembre de 2019) El precio de internet en el mundo. SIGLO XXI. Diario digital. https://bit.ly/2TMNaI1

Siteground. (2020). ¿Qué es el scraping de datos y cómo puedo detenerlo?

https://www.siteground.es/kb/scraping-datos/

TYSMagazine. (2015). La realidad aumentada y el turismo. https://bit.ly/3gzluA8

We are social. (2020).Global-Digital-Report-2019@ Wearesocial.Com.

https://wearesocial.com/global-digital-report-2019 